\title{
Periodontal ligament influence on the stress distribution in a removable partial denture supported by implant: a finite element analysis
}

Carlos Marcelo ARCHANGELO' ${ }^{1}$, Eduardo Passos ROCHA², João Antônio PEREIRA², Manoel MARTIN JUNIOR', Rodolfo Bruniera ANCHIETA ${ }^{4}$, Amilcar Chagas FREITAS JÚNIOR ${ }^{4}$

\author{
1-DDS, PhD, Professor, Federal Institute of Paraná - IFPR, Londrina, PR, Brazil. \\ 2-DDS, PhD, Associate Professor, Department of Dental Materials and Prosthodontics, Faculty of Dentistry of Araçatuba, UNESP - Univ. Estadual Paulista, \\ Araçatuba, SP, Brazil. \\ 3-MEng, PhD, Associate Professor, Department of Mechanical Engineering, Faculty of Engineering of Ilha Solteira, UNESP - Univ. Estadual Paulista, Ilha \\ Solteira, SP, Brazil. \\ 4-DDS, MS, PhD student, Department of Dental Materials and Prosthodontics, Faculty of Dentistry of Araçatuba, UNESP - Univ. Estadual Paulista, Araçatuba, \\ SP, Brazil.
}

Corresponding address: Prof. Adj. Dr. Carlos Marcelo Archangelo - Rua José Bonifacio, 1193, Vila Mendonça - Araçatuba - SP - Brasil - $16010-050$ - Phone: 55-18-36363290 - e-mail: carlosarchangelo@uol.com.br

Received: October 18, 2010 - Modification: August 7, 2011 - Accepted: September 1, 2011

\section{ABSTRACT}

\begin{abstract}
$\mathrm{O}$ bjective: The non-homogenous aspect of periodontal ligament (PDL) has been examined using finite element analysis (FEA) to better simulate PDL behavior. The aim of this study was to assess, by 2-D FEA, the influence of non-homogenous PDL on the stress distribution when the free-end saddle removable partial denture (RPD) is partially supported by an osseointegrated implant. Material and Methods: Six finite element (FE) models of a partially edentulous mandible were created to represent two types of PDL (non-homogenous and homogenous) and two types of RPD (conventional RPD, supported by tooth and fibromucosa; and modified RPD, supported by tooth and implant [10.00×3.75 mm]). Two additional FE models without RPD were used as control models. The non-homogenous PDL was modeled using beam elements to simulate the crest, horizontal, oblique and apical fibers. The load ( $50 \mathrm{~N}$ ) was applied in each cusp simultaneously. Regarding boundary conditions the border of alveolar ridge was fixed along the $x$ axis. The FE software (Ansys $10.0)$ was used to compute the stress fields, and the von Mises stress criterion ( $\sigma \mathrm{VM})$ was applied to analyze the results. Results: The peak of $\sigma \mathrm{VM}$ in non-homogenous PDL was higher than that for the homogenous condition. The benefits of implants were enhanced for the non-homogenous PDL condition, with drastic $\sigma \mathrm{VM}$ reduction on the posterior half of the alveolar ridge. The implant did not reduce the stress on the support tooth for both PDL conditions. Conclusion: The PDL modeled in the non-homogeneous form increased the benefits of the osseointegrated implant in comparison with the homogeneous condition. Using the non-homogenous PDL, the presence of osseointegrated implant did not reduce the stress on the supporting tooth.
\end{abstract}

Key words: Removable partial denture. Dental implant. Finite element analysis. Periodontal ligament.

\section{INTRODUCTION}

Conventional removable partial dentures (RPD) show a complex biomechanical behavior, mainly when the RPD is supported by teeth and fibromucosa, such as in Kennedy Class $\mathrm{I}^{25}$. This behavior has been better controlled when an osseointegrated implant is placed and acts as an additional support, retainer, or both, improving the stability and retention of the RPD, which reduces the demand on the support structures ${ }^{14}$.

In this context, the use of RPD and implants has been presented in a series of clinical possibilities that reflect individual experiences with some 
singularities according to the number and position of the remaining teeth in the arch, as well as the size of the prosthetic space and degree of bone loss ${ }^{2-4,9-}$ $11,13,14,16,17,20,26,28$. The usual clinical responses from patients are represented by better RPD stability, retention and comfort when this association is done ${ }^{14}$.

Mitrani, et al. ${ }^{18}(1993)$ investigated the free-end saddle RPD combined with an implant on the distal extension wearers for a mean period of 2.5 years. Those authors showed that the implant placed on the distal extension of RPD increases patient satisfaction, reduces the marginal bone loss, and establishes the peri-implant tissues ${ }^{18}$. Nonetheless, data about the abutment tooth behavior over time are still not reported and remain inconclusive.

Finite element (FE) studies can provide valuable information about the stress reduction on the support structures promoted by implants. However this information is controversial because the benefits of the osseointegrated implant are more evident on the alveolar ridge and hardly conclusive for the RPD abutment tooth ${ }^{23}$.

There are some concerns about the tooth behavior in a RPD supported by implant and tooth through finite element analysis (FEA), mainly because the periodontal ligament (PDL) has not been properly modeled as a non-homogenous structure. The homogenous aspect of the PDL in FEA might negatively change the results. Atmaram and Mohammed $^{1}$ (1981) reported that the homogeneous condition of the PDL is closer to more uniform stress distribution and unable to direct the forces applied in accordance with the set of fibers in vivo. In addition, the magnitude of stresses is shown to be less pronounced than under the non-homogeneous condition. This is particularly important in face of forces with non-axial incidence, producing terminal torqueing forces against the abutment teeth and the soft tissue ${ }^{19}$.

Other authors have pointed out that the nonhomogeneous PDL was necessary in order to understand how occlusal loads are absorbed by the $\mathrm{PDL}^{22}$. Therefore, this condition is decisive to analyze the results of RPD associated with an osseointegrated implant by FEA. Nevertheless, the benefits of the combination between RPD and osseointegrated implant may be analyzed from this perspective. The analysis of the stress distribution in the studies by Atmaram and Mohammed ${ }^{1}$ (1981) and Rees and Jacobsen ${ }^{22}$ (1997) did not show the behavior of the abutment tooth.

The objective of this study was to evaluate the influence of PDL condition (homogenous and nonhomogenous) on the stress distribution of a freeend saddle RPD associated with an osseointegrated implant through 2-D FEA. The hypothesis tested was that non-homogeneous PDL increases the benefits of implant, reducing the stress on the supporting tooth of the RPD supported by tooth and implant.

\section{MATERIAL AND METHODS}

Six mandibular models ( A, B, C, D, E, and F) were modeled using graphic software (AutoCAD, Autodesk Inc., San Rafael, CA, USA). In a sagittal view, all models simulated partially edentulous hemi-arches without posterior dental support (Kennedy Class I). In addition, all models had the remaining tooth 33 and a distal extension ridge.

The characteristics of the abutment - the length of the distal extension ridge, the dimensions and characteristics of the support and protective periodontium, the mandibular bone height, the thickness of the $\mathrm{CoCr}$ metal structure, and the number of artificial teeth - were kept constant. The difference was the PDL in models D, E, and F, which was reproduced in accordance with the in vivo characteristic, with representation of the following 4 groups of fibers: crest, horizontal, oblique and apical (Figure 1).

After the models had been created, the files were exported to ANSYS 8.0 (Swanson Analysis Systems, Houston, PA, USA) to discriminate the regions and generate the FE mesh. Models A, B, and C, (Figure 1) were considered homogeneous, isotropic, and linearly elastic. The mechanical properties adopted for all materials (elastic modulus and Poisson's ratio) were established according to the literature (Table 1) 1,8,15,23,24,29.

Models D, E, and MF, similar to models A, B, and $C$, respectively, were considered homogeneous, isotropic, and linearly elastic, except for the PDL, which was considered a non-homogeneous structure. Thus, models D, E, and $F$ differed from models $A, B$ and $C$ only by their structural characteristic of the PDL, being similar in all other factors.

The element used to generate the mesh was the plane 2 element, a triangular element defined by 6 nodes, with 2 degrees of freedom per node, and quadratic displacement behavior. The FE mesh showed up to 80,000 nodes and up to 40,000 elements. This configuration allowed the appropriate refinement of the mesh in thin structures, such as the cortical bone and the PDL, reaching the convergence norm.

As far as the creation of the non-homogeneous PDL is concern, using the methodology established by Atmaram and Mohammed ${ }^{1}$ (1981) with some modifications, the composition of the PDL in the FE model was established under the in vivo condition ${ }^{5}$. For this, beam elements were used to represent 4 groups of PDL fibers in a sagittal cut (crest, horizontal, oblique and apical fibers) and their directions. To support the bending movements, as 
well as tensile and compression loads, the crosssectional area and the moment of inertia for the beam elements were used to describe the PDL. Thus, the following equations were used:

Cross sectional area: $A=n \times(r)^{2}$

Moment of inertia: $I=n \times(d)^{4} / 64$

where:

$A=$ cross sectional area; $I=$ moment of inertia; $\Pi=3.14 ; d=4 \mu \mathrm{m}$ or $4 \times 10^{-6} \mathrm{~m} ; \mathrm{r}=2 \times 10^{-6} \mathrm{~m}$.
Grant, et al. ${ }^{12}$ (1988) determined a value of 4 $\mu \mathrm{m}$ for the PDL fibers of an RPD abutment tooth. Thus, the values found for the cross-sectional area and the moments of inertia of each element were $1,256 \times 10^{-5} \mathrm{~mm}^{2}$ and $0.785 \times 10^{-12} \mathrm{~mm}^{4}$, respectively.

In accordance with that established by Atmaram and Mohammed ${ }^{1}$ (1981), the number of periodontal fibers adopted under the non-homogeneous condition was half the number of elements present

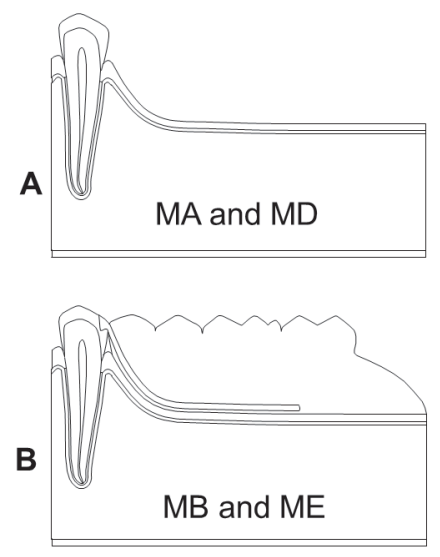

$\mathbf{L}$ 创

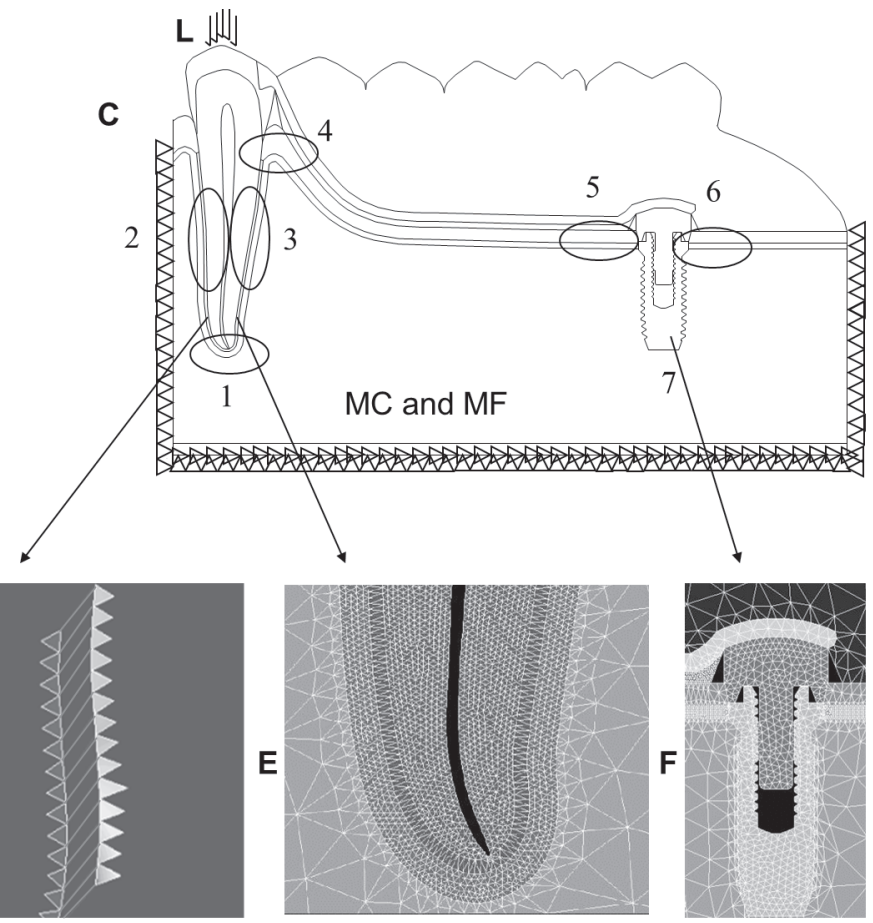

Figure 1- Description of finite element models, finite element mesh and boundary condition. A: Models A, B, and C. B Model B with the removable partial denture (RPD). C: Model C with RPD and implant. Areas (1 to 6) selected to perform the analysis in all models. D:- The beam elements used to build the non-homogeneous periodontal ligament (PDL). E and F: Mesh refinements for tooth apex and implant, respectively. $L$ shows the distributed loading scheme. The entire right and left sides were fixed on the $x$ axis. The bone at the bottom of the model was fixed on the $x$ and $y$ axes

Table 1- Mechanical properties of the materials. E - Elastic modulus. v - Poisson's ratio. PDL - Periodontal ligament. Ti - Titanium

\begin{tabular}{lcccc}
\hline \multicolumn{1}{c}{ Structures } & E (GPa) & References & $\mathbf{v}$ & References \\
\hline Enamel & 41 & 17 & 0.3 & 17 \\
Dentin & 18.6 & 18 & 0.31 & 18 \\
\hline Homogenous PDL & 0.175 & 15 & 0.45 & 15 \\
Non-homogenou PDL & 0.35 & 15 & 0.45 & 15 \\
\hline Fibromucosa & 0.68 & 17 & 0.45 & 17 \\
Cortical bone & 13.7 & 18 & 0.3 & 18 \\
Medullar bone & 1.37 & 18 & 0.3 & 18 \\
Implant (Ti) & 103.4 & 20 & 0.35 & 20 \\
\hline Healing abutment $(\mathrm{Ti})$ & 103.4 & 20 & 0.35 & 20 \\
CoCr cast alloy & 185 & 21 & 0.35 & 21 \\
Acrylic resin & 8.3 & 14 & 0.28 & 14 \\
Artificial teeth & 8.3 & 14 & 0.28 & 14 \\
\hline
\end{tabular}


in the PDL under the homogeneous condition. Thus, all models with a homogeneous PDL $(A, B$, and $C)$ contained 824 elements in the PDL region, and the models with a non-homogeneous PDL ( $D, E$, and F) contained 412 fibers for composition of the PDL.

As the boundary condition, the left and right sides of the models were fixed only in the $x$ direction, in order to prevent lateral movement of the structures. This allowed the vertical movement of the RPD base over the fibromucosa and, consequently, the deformation of the cortical and medullar bone beneath it, as well as simulation of the bilateral mandibular condition. Only the cortical bone at the base of the models was fixed in the $x$ and y directions (Figure 1).

The models were loaded with vertical forces (50
N) distributed at each cusp, fractionated into 5 point loads of $10 \mathrm{~N}$ each to prevent the occlusal contact characterized by only one point load (Figure 1 ).

The von Mises equivalent stress criterion $\left(\sigma_{\mathrm{vM}}\right)$ was adopted to analyze the stress distribution. Each structure was individually analyzed following specific areas (Figure 1), as well as to allow the data to be refined into regions of interest, as follows:

Area 1: Root apex; Area 2: Mesial side of the abutment tooth and adjacent structures; Area 3: Distal side of the abutment tooth and adjacent structures; Area 4: Distal bone crest of the abutment tooth; Area 5: Anterior half of the alveolar ridge; Area 6: Posterior half of the alveolar ridge; Area 7: Osseointegrated implant.

For more details and to allow the comparison

Table 2- von Mises stress $\left(\sigma_{\mathrm{vM}}\right)$, in MPa, for the cortical bone according to specific areas in models A to $\mathrm{F}$. Note the subdivision of regions 2 and 3 into 10 segments ( 1 to 10) around the abutment tooth

\begin{tabular}{|c|c|c|c|c|c|c|c|c|c|c|c|}
\hline \multirow[b]{2}{*}{ Models } & \multirow[b]{2}{*}{ Areas } & \multicolumn{10}{|c|}{ Subdivision of the regions 2 and 3 around the abutment tooth } \\
\hline & & 1 & 2 & 3 & 4 & 5 & 6 & 7 & 8 & 9 & 10 \\
\hline \multirow[t]{6}{*}{ A } & 1 & \multicolumn{10}{|c|}{10.5} \\
\hline & 2 & 10.5 & 10.5 & 12.2 & 12.2 & 14 & 15.7 & 17.5 & 19.2 & 21 & 19.2 \\
\hline & 3 & 8.7 & 5.2 & 5.2 & 7 & 7 & 8.7 & 8.7 & 10.5 & 12.2 & 19.2 \\
\hline & 4 & \multicolumn{10}{|c|}{8.7} \\
\hline & 5 & \multicolumn{10}{|c|}{3.5} \\
\hline & 6 & \multicolumn{10}{|c|}{1.7} \\
\hline \multirow[t]{6}{*}{ B } & 1 & \multicolumn{10}{|c|}{33.4} \\
\hline & 2 & 14.3 & 14.3 & 20.7 & 20.7 & 20.7 & 27 & 27 & 27 & 27 & 20.7 \\
\hline & 3 & 20.7 & 20.7 & 27 & 27 & 27 & 33.4 & 33.4 & 39.7 & 77.8 & 77.8 \\
\hline & 4 & \multicolumn{10}{|c|}{27} \\
\hline & 5 & \multicolumn{10}{|c|}{20.7} \\
\hline & 6 & \multicolumn{10}{|c|}{14.3} \\
\hline \multirow[t]{6}{*}{ C } & 1 & \multicolumn{10}{|c|}{25.1} \\
\hline & 2 & 25.1 & 12.9 & 19 & 19 & 25.1 & 25.1 & 31.2 & 31.2 & 31.2 & 19 \\
\hline & 3 & 25.1 & 19 & 25.1 & 25.1 & 25.1 & 31.2 & 37.4 & 43.5 & 74 & 74 \\
\hline & 4 & \multicolumn{10}{|c|}{25.1} \\
\hline & 5 & \multirow{2}{*}{\multicolumn{10}{|c|}{$\begin{array}{c}19 \\
12.9\end{array}$}} \\
\hline & 6 & & & & & 12 & & & & & \\
\hline \multirow[t]{6}{*}{ D } & 1 & \multicolumn{10}{|c|}{8.6} \\
\hline & 2 & 103 & 42.9 & 42.9 & 42.9 & 42.9 & 42.9 & 42.9 & 42.9 & 51.2 & 51.2 \\
\hline & 3 & 77.2 & 17.2 & 8.6 & 8.6 & 8.6 & 8.6 & 8.6 & 8.6 & 17.2 & 25.7 \\
\hline & 4 & \multicolumn{10}{|c|}{77.2} \\
\hline & 5 & \multicolumn{10}{|c|}{17.2} \\
\hline & 6 & \multicolumn{10}{|c|}{8.6} \\
\hline \multirow[t]{6}{*}{$E$} & 1 & \multicolumn{10}{|c|}{34.8} \\
\hline & 2 & 86.3 & 43.4 & 34.8 & 34.8 & 34.8 & 34.8 & 34.8 & 34.8 & 34.8 & 34.8 \\
\hline & 3 & 86.3 & 52 & 43.4 & 52 & 52 & 52 & 52 & 60.6 & 103.5 & 103.5 \\
\hline & 4 & & & & & 86 & & & & & \\
\hline & 5 & & & & & 26 & & & & & \\
\hline & 6 & & & & & 17 & & & & & \\
\hline $\mathrm{F}$ & 1 & & & & & & & & & & \\
\hline & 2 & 91.6 & 41.8 & 41.8 & 41.8 & 41.8 & 41.8 & 41.8 & 33.5 & 33.5 & 25.2 \\
\hline & 3 & 83.3 & 50.1 & 50.1 & 50.1 & 50.1 & 50.1 & 50.1 & 58.4 & 100 & 100 \\
\hline & 4 & & & & & 83 & & & & & \\
\hline & 5 & & & & & 25 & & & & & \\
\hline & 6 & & & & & & & & & & \\
\hline
\end{tabular}


Table 3- von Mises stress $\left(\sigma_{\mathrm{vM}}\right)$, in MPa, for the medullar bone according to specific areas (1 to 6 ) in the models (A to F)

\begin{tabular}{ccccccc}
\hline & \multicolumn{7}{c}{ Areas } & $\mathbf{5}$ & $\mathbf{6}$ \\
\hline Models & $\mathbf{1}$ & $\mathbf{2}$ & $\mathbf{3}$ & $\mathbf{4}$ & $\mathbf{5}$ & 0.7 \\
A & 8.3 & 2.8 & 2.8 & 1.4 & 0.7 & 9.3 \\
B & 17.8 & 5.1 & 5.1 & 3.7 & 8.3 & 4.4 \\
C & 18.1 & 4.4 & 6.4 & 4.4 & 1.9 & 1.9 \\
D & 11.4 & 5.7 & 1.9 & 3.8 & 10.2 & 7.3 \\
E & 17.4 & 4.4 & 5.8 & 11.6 & 11.9 & 4.3 \\
\hline F & 17.6 & 4.9 & 6.1 & 11.8 & & \\
\hline
\end{tabular}

Table 4- von Mises stress ( $\left.\sigma_{\mathrm{vM}}\right)$, in MPa, for the fibromucosa according to specific areas (4 to 6 ) in the models (A to F)

\begin{tabular}{cccc}
\hline & \multicolumn{3}{c}{ Areas } \\
Models & $\mathbf{4}$ & $\mathbf{5}$ & $\mathbf{6}$ \\
\hline A & 5.7 & 0.9 & 0.9 \\
B & 7 & 8.6 & 7.8 \\
C & 7.1 & 8 & 3.7 \\
D & 95 & 8.3 & 8.3 \\
E & 59.7 & 14.1 & 14.1 \\
F & 61.3 & 13.7 & 6.9 \\
\hline
\end{tabular}

with the study of Atmaram and Mohamed $^{1}$ (1981), the previously determined areas 2 and 3 were subdivided into 10 parts.

\section{RESULTS}

\section{Cortical bone}

For the homogeneous PDL, the stress concentration on the cortical bone around tooth 33 gradually increased in the bone crest (tooth apex direction) in areas 2 and 3 of models A, B, and C. The presence of the conventional RPD (model $B$ ) drastically increased the stress on areas 5 and 6 in comparison with MA, and the presence of the osseointegrated implant (model C) reduced the $\sigma_{\mathrm{vM}}$ on the posterior half of the ridge, mainly in area 6 (Table 2).

For the non-homogeneous PDL (models D, E, and $F)$, the svM in areas 2 and 3 of model D diminished in the bone crest (root apex direction). The presence of the conventional RPD (model E) increased the $\sigma_{\mathrm{vM}}$ in areas $1,4,5$, and 6 compared with model $D$, and the presence of the osseointegrated implant (model F) drastically reduced the stresses in area 6 of the alveolar ridge (Table 2).

\section{Medullar bone}

The incorporation of the RPD (model B) drastically increased the $\sigma_{\mathrm{vM}}$ on the trabecular bone for areas 5 and 6 by approximately $1235 \%$ and $834 \%$, respectively, when compared with the respective areas of model $\mathrm{A}$ (Table 3 ). The presence of the osseointegrated implant (model $\mathrm{C}$ ) reduced the $\sigma_{\mathrm{vm}}$ in those areas, similar to what occurred in the cortical bone.

For the non-homogeneous PDL, model E increased the $\sigma_{v M}$ in areas 5 and 6 (Table 3) compared with areas 5 and 6 of model B for the homogeneous PDL. The osseointegrated implant (model F) also reduced the stress levels in area 6 of the ridge (Table 3 ).

\section{Fibromucosa}

The high $\sigma_{\mathrm{vM}}$ in areas 5 and 6 of the fibromucosa in model $B$, corroborate those found for the same cortical and trabecular bone areas in relation to the homogeneous PDL. The osseointegrated implant (model C) also provided the fibromucosa with stress relief in areas 5 and 6 of the ridge, when compared with the $\sigma_{\mathrm{vM}}$ of model B (Table 4).

For the PDL under the non-homogeneous condition in areas 5 and 6 , the RPD (model E) increased the $\sigma_{v M}$, however, in lower ratio than those observed under the homogeneous condition, approximately $69 \%$ in both areas. The osseointegrated implant also provided model $F$ with a reduction in $\sigma_{v M}$ in areas 5 and 6 of the fibromucosa (Table 4).

\section{Implant}

The osseointegrated implant showed a similar behavior for the homogeneous and nonhomogeneous PDL conditions. The pitch of the internal threads of the implants was responsible for the high $\sigma_{\mathrm{vM}}$ in models $\mathrm{C}$ and $\mathrm{F}$.

\section{DISCUSSION}

The conventional RPD applies moment of force or binary forces on the abutment tooth and alveolar ridge $e^{6,7}$. The associations of the RPD with an osseointegrated implant aims to increase the retention and stability, as well as provide a reduction of the stress on the support tooth, fibromucosa and alveolar ridge ${ }^{14}$.

In a previous FEA study with the RPD and 
implant ${ }^{23}$, this reduction of stress on the support tooth was not confirmed ${ }^{23}$. It was considered that the homogeneous PDL condition might reduce the stress in the alveolar ridge, but was not efficient in reducing the stress on the abutment tooth when an osseointegrated implant was distally supporting the acrylic resin base of the RPD.

In the present study, when the homogeneous PDL condition was simulated, the stress concentration on the cortical and medullar bone remained high at the abutment tooth apex. The $\sigma_{\mathrm{vM}}$ increased gradually in the bone crest - tooth apex direction in areas 2 and 3 (models A, B, and C). The greatest benefit of the osseointegrated implant was the reduction of the stresses on the posterior half of the alveolar ridge. The peak stress in the implant was observed on the neck closer to the cortical bone.

In agreement with another study ${ }^{3}$, the higher $\sigma_{v M}$ found in the medullar bone for the homogenous PDL condition occurred at the apex of the osseointegrated implant. It is pointed out that the stress found on the cortical bone around the osseointegrated implant remained below the stress levels found at the abutment tooth apex, showing that RPD associated with an osseointegrated implant to be a feasible and safe alternative, according Mitrani, et al. ${ }^{18}$ (2003).

Another benefit of the implant (model C) was the stress reduction in the fibromucosa in the posterior half compared with the model $B$. The implant provided anchorage for the acrylic resin base of the RPD, limiting its vertical intrusion movement and reducing the $\sigma_{\mathrm{vM}}$ when it was compared with the model $B$.

Nevertheless, the use of the non-homogeneous PDL (models D, E, and F) changed the stress distribution pattern in practically all regions analyzed. The peak of stress was higher for almost all structures with the non-homogenous condition than with the homogeneous condition (models $A$, $B$, and $C$ ). However, the stress variation around the abutment tooth was lower compared with models $D, E$, and $F$, and its distribution was similar to that established by others authors $1,21,27$.

The modeling type for PDL has a significant effect on the nature and magnitude of the alveolar stress $^{1,21,27}$. It occurs because the modeling of homogeneous PDL is like an assumption of a hypothetical soft interactive medium in which the stress is shared uniformly; hence, it uniformly distributes the stresses to bone. In contrast, in the heterogeneous PDL assumption with the main fibers of the PDL incorporated, there is no direct contact between the individual fibers, and the stresses among the fibers are not shared equally, resulting in the wide variation of the stresses in the adjoining structure, such as the cortical bone, reproducing more accurately the events of the PDL in function.
Similarly to Atmaram and Mohammed ${ }^{1}$ (1981), in the present study there was an inversion in the direction of stress, since the stresses around tooth 33 gradually diminished in the direction of the bone crest to the tooth apex when the non-homogeneous PDL was simulated. Furthermore, the increase of $\sigma_{\mathrm{vM}}$ on the anterior and posterior halves of the alveolar ridge for areas 5 and 6 (Table 4) were accentuated in model B. A similar behavior was not verified in models $D$ and $E$, as the $\sigma_{v M}$ increased $69.1 \%$ and $69.1 \%$ for those areas, respectively.

The results found in models $A, B$, and $C$, with the homogeneous PDL condition, might be not represent the potentially artificial, since the peak of $\sigma_{\mathrm{vM}}$, as well the stress variation was not repeated in the equivalent models, D, E, and F, respectively. In addition, the stress around the abutment tooth, and in the posterior area of the alveolar ridge in models $\mathrm{C}$ and $\mathrm{F}$, should be closer to that observed in models $A$ and $D$, with no RPD, respectively. It was observed that the result closest to this hypothesis was showed by the posterior half of the fibromucosa. Even with the use of non-homogeneous PDL, the stress variation around the abutment tooth in models $E$ and $F$ was small, rejecting the hypothesis of the present study. This aspect partially supports the results pointed by Keltjens, et al. ${ }^{14}$ (1993) that the osseointegrated implant does not reduce the stress on the abutment tooth.

The results of the present study keep valid the data from Atmaram and Mohammed ${ }^{1}$ (1981) because the direction of demand on the abutment tooth and the behavior of the bone showed correlation with the experiment ${ }^{1}$. This aspect reinforces the condition that the PDL used in its non-homogeneous form appropriately reproduces the PDL in function ${ }^{21,27}$.

The main benefit of the osseointegrated implant placed in the position as performed in the present study is reducing the stress on the fibromucosa and alveolar ridge. These benefits are more evident when the PDL is modeled in its non-homogenous form.

\section{CONCLUSION}

The PDL modeled in the non-homogeneous form increases the benefits of the osseointegrated implant in comparison with the homogeneous condition. Using the non-homogenous PDL, the presence of osseointegrated implant did not reduce the stress on the supporting tooth. 


\section{REFERENCES}

1- Atmaram GH, Mohammed H. Estimation of physiologic stresses with a natural tooth considering fibrous PDL structure. ] Dent Rest. $1981 ; 60: 873-7$.

2- Attard NJ, Zarb GA. Long-term treatment outcomes in edentulous patients with implant-fixed prostheses: the Toronto study. Int J Prosthodont. 2004;17:417-24.

3- Barbier L, Sloten LV, Krzesinski G, Schepers E, Van Der Perre G. Finite element analysis of non-axial versus axial loading of oral implants in the mandible of the dog. J Oral Rehabil. 1998;25:84758.

4- Battistuzzi PGFCM, Van Slooten H, Käyser AF. Management of an anterior defect with a removable partial denture supported by implants and residual teeth: a case report. Int J Oral Maxillofac Implants. 1992; 7:112-5.

5- Berkovitz BKB, Moxham BJ, Newman HN. The periodontal ligament in health and disease. $2^{\text {nd }}$ ed. London: Ed.Mosby- Wolfe; 1995. $446 \mathrm{p}$.

6- Chou TM, Caputo AA, Moore JD, Xiao B. Photoelastic analysis and comparison of force-transmission characteristics of intracoronal attachments with clasp distal-extension removible partial dentures. J Prosthet Dent. 1989;62:313-9.

7- Chou TM, Eick JD, Moore DJ, Tira DE. Stereophotogrammetric analysis of abutment tooth movement in distal-extension removable partial dentures with intracoronal attachements and clasps. J Prosthet Dent. 1991;66:343-9.

8- Farah JW, Craig RG, Meroueh KA. Finite element analysis of a mandibular model. J Oral Rehabil. 1988;15:615-24.

9- Ganz SD. Combination natural tooth and implant-borne removable partial denture: a clinical report. J Prosthet Dent. 1991;66:1-5.

10- George MA. Removable partial denture design assisted by osseointegrated implants. J Calif Dent Assoc. 1992;20:64-6.

11- Giffin KM. Solving the distal extension removable partial denture base movement dilemma: a clinical report. J Prosthet Dent. 1996;76:347-9.

12- Grant DA, Stern IB, Listgarten MA. Periodontics. $6^{\text {th }}$ ed. St. Louis: Mosby; 1988. 1154 p.

13- Jang Y, Emtiaz S, Tarnow DP. Single implant-supported crown used as an abutment for a removable cast partial denture: a case report. Implant Dent. 1998; 7:199-204.

14- Keltjens HMAM, Käyser AF, Hertel R, Battistuzzi PGF. Distal extension removable partial dentures supported by implants and residual teeth: considerations and case reports. Int J Oral Maxillofac Implants. 1993;8:208-13.
15- Ko CC, Chu CS Chung KH, Lee MC. Effects of posts on dentin stress distribution in pulpless teeth. J Prosthet Dent. 1992;68:421-7.

16- McAndrew R. Prosthodontic rehabilitation with a swing-lock removable partial denture and a single osseointegrated implant: a clinical report. J Prosthet Dent. 2002;88:128-31.

17- Mijiritsky E and Karas S. Removable partial denture design involving teeth and implants as an alternative to unsuccesful fixed implant therapy: a case report. Implant Dent. 2004;13:218-22. 18- Mitrani R, Brudvik JS, Phillips KM. Posterior implants for distal extension removable prostheses: a retrospective study. Int J Periodontics Restorative Dent. 2003;23:353-9.

19- Ohkubo C, Kobayashi M, Suzuki Y, Hosoi T. Effect of implant support on distal-extension removable partial dentures: in vivo assessment. Int J Oral Maxillofac Implants. 2008;23:1095-101.

20- Pellecchia M, Pellecchia R, Emtiaz, S. Distal extension mandibular removable partial denture connected to an anterior fixed implant-supported prosthesis: a clinical report. J Prosthet Dent. 2000;83:607-12.

21- Qian H, Chen J, Katona TR. The influence of the PDL principal fibers in a 3-dimensional analysis of orthodontic tooth movement. Am J Orthod Dentofacial Orthop. 2001;120:272-9.

22- Rees JS, Jacobsen PH. Elastic modulus of the periodontal ligament. Biomaterials. 1997;18:995-9.

23- Rocha EP, Luersen MA, Pellizzer EP, Del Bel Cury AA. Distal-extension removible partial denture associated with an osseointegrated implant. Study by finite element method [Abstract. No. 1948]. ] Dent Res. 2003;82:254.

24- Sertgoz A, Gunever S. Finite element analysis of the effect of cantilever and implant length on stress distribution in an implantsupported fixed prosthesis. J Prosthet Dent. 1996;76:165-9.

25- Shahmiri RA, Atieh MA. Mandibular Kennedy Class I implanttooth-borne removable partial denture: a systematic review. J Oral Rehabil. 2010;37:225-34.

26- Starr NL. The distal extension case: an alternative restorative design for implant prosthetics. Int J Periodontics Restorative Dent. 2001;21:61-7.

27- Toms SR, Eberhardt AW. A nonlinear finite element analysis of the periodontal ligament under orthodontic tooth loading. Am J Orthod Dentofacial Orthop. 2003;123:657-65.

28- Uludag B, Celik G. Fabrication of a maxillary implant-supported removable partial denture: a clinical report. J Prosthet Dent. 2006;95:19-21.

29- Williams DF. Biocompatibility of clinical implant materials. Boca Raton: CRC Press; 1981. p. 99-127. 